\title{
Réactions Cellulaires des muQUeUses nasales et SinUsales DES CHÈVRES ET DES MOUTONS À L'INFESTATION NATURELLE par Oestrus ovis Linné 1758 (Diptera: Oestridés)
}

\author{
NGUYEN VAN-KHANH*, JACQUIET P.*, DURANTON C.*, BERGEAUD J.P.*, PREVOT F.* \& DORCHIES P.*
}

Summary : MUCOUS CELLULAR RESPONSES OF SHEEP AND GOATS Naturally infected by CEstrus ovis linNé 1758 (Diptera: Cestridae) CEstrosis is a very common myiasis of sheep and goats in meditterranean and tropical countries. Goats are suitable hosts for OEstrus ovis but the parasitic burden remain lower than in sheep. Cellular responses (mucous and serous mast cells, eosinophils and globules leucocytes) were measured in 30 infected and 30 non infected sheep and in 23 infected and 24 non infected goats. The presence of OEstrus ovis larvae led to an important inflammatory response in sheep and in goats as well. Furthermore, the intensity of the cellular response correlated with the larval burden, specially with globules leucocytes and eosinophils. Nevertheless, huge differences occured between sheep and goats responses even in similar larval burden range. Infected sheep showed larger counts of mucous mast cells than goats, the differences were smaller in serous mast cells and eosinophils and no difference was detected in globules leucocytes (intraepithelial mast cells) counts between the two hosts species. These results were compared with those obtained in gastro-intestinal strongyles infections.

KEY WORDS : œestrosis, œestrus ovis, cellular response, goat, sheep, mast cell, eosinophil, globule leucocyte.

MOTS CLÉS : œstrose, œstrus ovis, réaction cellulaire, chèvre, mouton, mastocyte, eosinophiles, globule leucocyte.

\section{Résumé :}

L'œstrose est une myiase naso-sinusale du mouton et de la chèvre causée par les larves d'un Diptère parasite obligatoire, OEstrus ovis, particulièrement fréquente dans les pays méditerranéens et tropicaux. La chèvre, tout en permettant un développement total du parasite, est moins souvent et moins massivement infestée que le mouton. Les réponses cellulaires en mastocytes muqueux et séreux, en éosinophiles et en globules leucocytes des muqueuses nasales et sinusales de 60 moutons (30 infestés et 30 indemnes) et de 47 chèvres (23 infestées et 24 non infestées) ont été mesurées et analysées avec des méthodes statistiques multivariées. La présence de larves d'OEstrus ovis s'accompagne chez le mouton comme chez la chèvre d'un recrutement local très important de cellules inflammatoires. L'intensité de cette réponse cellulaire est corrélée au nombre de larves présentes sur les muqueuses notamment pour les globules leucocytes (mastocytes intraépithéliaux) et les éosinophiles. Enfin, des différences importantes existent entre les réponses cellulaires des chèvres et des moutons face à des infestations d'intensités égales. Les moutons infestés recrutent beaucoup plus de mastocytes muqueux que les chèvres infestées alors que les différences sont plus faibles pour les mastocytes séreux et les éosinophiles et qu'elles ne sont plus significatives pour les globules leucocytes. Ces résultats d'histopathologie obtenus dans le modèle d'œstrose ovine sont comparés à ceux obtenus lors d'infestations par les trichostrongles gastro-intestinaux.

\section{INTRODUCTION}

I 'œestrose est une myiase naso-sinusale du mouton et de la chèvre causée par les larves d'un diptère fréquente dans les pays méditerranéens et tropicaux (Belem \& Rouille, 1988; Bergeaud et al., 1994; Ogunrinade, 1977; Yilma \& Dorchies, 1991). Cette myiase s'exprime cliniquement par du jetage, des éternuements et de la sinusite, ces signes cliniques semblent toutefois être plus fréquents et plus accentués chez les moutons que chez les chèvres (Horak, 1977a, b). D'autre

\footnotetext{
* Laboratoire associé INRA de physiopathologie respiratoire des ruminants. URA 959, École Nationale Vétérinaire de Toulouse, 23, chemin des Capelles, F-31076 Toulouse Cédex 03

Correspondance : Philippe Jacquiet.

Tél. : 0561193967 - Fax : 0561193944.

E-mail: p.jacquiet@envt.fr
}

part, la présence des larves dans les cavités nasales et sinusales favorise l'apparition d'abcès pulmonaires et de pneumonie interstitielle (Dorchies et al., 1993) par un mécanisme qui reste inconnu.

La présence de larves d'O. ovis chez le mouton provoque une augmentation importante du nombre d'éosinophiles et de mastocytes dans le chorion sous épithélial des muqueuses du septum nasal, des cornets et des sinus (Jagannah et al., 1989; Nguyen van Khanh et al., 1996). La réponse éosinophilique est particulièrement massive chez les moutons parasités, les populations sont jusqu'à 60 fois supérieures à celles observées chez des agneaux sains (Nguyen van Khanh et al., op. cit.). Il existe cependant de grandes variations individuelles qui peuvent être en relation avec l'intensité de la charge parasitaire ou les capacités de réponse de l'hôte.

Jusqu'à présent, aucune étude de la réponse cellulaire à l'infestation par $O$. ovis n'a été réalisée chez la chèvre qui semble moins réceptive à ce parasite. En effet, la 
prévalence et l'intensité de l'infestation sont plus faibles chez les chèvres que chez les moutons (Horak, 1977a, b; Belem \& Rouille, 1988; Chlabra \& Ruprah, 1976).

Les objectifs de cette étude ont été : i) de comparer les populations cellulaires des moutons et des chèvres infestés ou non, ii) de rechercher une éventuelle corrélation entre l'intensité des réponses cellulaires et la charge parasitaire.

\section{MATÉRIELS ET MÉTHODES}

\section{ANIMAUX}

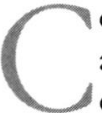
ette étude a été réalisée sur 47 chèvres de race alpine et 60 moutons (en l'occurence des brebis) plus, naturellement exposés à l'infestation par CEstrus ovis au cours de leur vie au pâturage. Les chèvres provenaient du département de la Drôme (abattage à l'abattoir municipal de Pézenas) tandis que les moutons étaient originaires du département de l'Ariège (abattage à l'abattoir de Pamiers). Tous les animaux ont été examinés en janvier ou février 1997 c'est-à-dire à une période de l'année où il n'y a pas d'infestations nouvelles et où $O$. ovis survit essentiellement sous forme de larves de premier stade inhibées chez ses hôtes. Après fente longitudinale de la tête, les larves ont été recherchées selon la technique décrite par Yilma \& Dorchies, 1991.

\section{PRÉLÈVEMENTS HISTOLOGIQUES}

Les prélèvements histologiques de la muqueuse respiratoire supérieure ont été faits dans trois sites anatomiques différents : septum, cornets et sinus frontal. Pour chacun de ces sites, deux échantillons d'environ $1 \mathrm{~cm}^{2}$ ont été réalisés côte à côte toujours dans la même zone durant toute l'étude pour tous les animaux. Un prélèvement a été fixé dans du formol à $10 \%$ pour la recherche des éosinophiles et des mastocytes intraépithéliaux (globule leucocytes des auteurs anglosaxons), et son homologue dans du fixateur de Carnoy pour l'identification des mastocytes muqueux et séreux.

\section{EXAMENS HISTOLOGIQUES}

Chaque prélèvement a été inclus selon les méthodes habituelles et des coupes de $2 \mu \mathrm{m}$ ont été réalisées avant coloration au May-Grunwald-Giemsa pour les éosinophiles (EO), au Carbol-chromotrope pour les globules leucocytes (GL), au Bleu de Toluidine pour les mastocytes séreux (MS) et au Bleu-Alcian Safranine pour les mastocytes muqueux (MM).

Les dénombrements cellulaires ont été faits au microscope photonique (Objectif $\times 50$ à immersion) dans trois zones sur chaque coupe : chorion sous-épithé- lial, chorion interglandulaire et sous muqueuse (10 champs optiques chacune) pour les éosinophiles et les mastocytes, dans l'épithélium (30 champs) pour les globules leucocytes. Les résultats des comptages des mastocytes et des éosinophiles sont exprimés par la somme des cellules comptées dans le chorion sous épithélial, le chorion interglandulaire et la sous muqueuse. Ils ont ensuite été exploités en tenant compte du type cellulaire identifié, du site anatomique (sinus, cornets ou septum nasaux) et du statut de l'animal : infesté ou indemne au moment de l'abattage. Ainsi pour chaque individu, douze variables " cellules " sont prises en compte : mastocytes séreux dans le sinus (SI MS), dans les cornets (CO MS) et dans le septum (SE MS), mastocytes muqueux dans les sinus (SI MM), dans les cornets (CO MM) et dans le septum (SE MM), éosinophiles dans le sinus (SI EO), dans les cornets (CO $\mathrm{EO}$ ) et dans le septum (SE EO), enfin, globules leucocytes dans les sinus (SI GL), les cornets (CO GL) et le septum (SE GL).

\section{ANALYSES STATISTIQUES}

Une transformation de la forme log $(x+1)$ a été effectuée sur les comptages bruts des différents types cellulaires, $x$ étant le total des comptages sur 30 champs optiques pour chaque localisation anatomique.

Comparaison animaux infestés et animaux indemnes Les réactions cellulaires des chèvres et des moutons indemnes ont été comparées respectivement aux réactions cellulaires des chèvres et des moutons parasités par une analyse de variance et par une analyse factorielle discriminante (AFD) au moyen du logiciel StatItcf. L'AFD a été réalisée en prenant en compte les douze variables " cellules ". Ce type d'analyse permet de mieux séparer les groupes (infestés versus non infestés) par une fonction discriminante (combinaison linéaire des douze variables qui optimise la variance inter-groupe et minimise la variance intra-groupe). Elle classe ou affecte également les individus selon que leur profil cellulaire est plus proche du profil moyen des infestés ou de celui des non infestés (classement des individus dans un tableau d'appartenance).

Différences de réaction cellulaire entre moutons et chèvres

Les réponses cellulaires des chèvres et des moutons ont été comparées au moyen d'une AFD dans le cas des animaux indemnes d'une part et dans le cas des animaux parasités d'autre part.

Relation entre l'intensité de la réponse cellulaire et le nombre de larves présentes

Les variables quantitatives (numérations cellulaires et nombre de larves) ont été transformées en variables qualitatives équilibrées (légende de la figure 1) et une 
analyse factorielle des correspondances multiples (AFCM) a été réalisée pour les chèvres d'une part et pour les brebis d'autre part. Les variables étudiées en ACM sont projetées sur un plan. Chacun des axes 1 et 2 est une combinaison linéaire de ces variables et rend compte de la variabilité totale. La proximité de deux variables sur un plan suggère qu'elles sont corrélées. Enfin, après transformation $\log (\mathrm{x}+1)$, les données chèvres et moutons ont été analysées en régression multiple progressive au moyen du logiciel Stat-Itcf.

\section{RÉSULTATS}

\section{POPULATIONS PARASITAIRES}

T es dénombrements larvaires et l'identification des différents stades larvaires observés sont présentés dans le tableau I. Parmi les chèvres infestées, $65,2 \%$ l'étaient par une à cinq larves, 30,41\% par six à 15 larves et 4,39\% par plus de 16 larves tandis que chez les moutons 26,7\% étaient infestés par une à cinq larves, 33,3\% par six à 15 larves et $40 \%$ par plus de 16 larves.

On retrouve une grande majorité de larves de premier stade (stade en hypobiose) chez les moutons comme chez les chèvres. La structure démographique des populations d' Estrus ovis (larves en hypobiose versus larves en développement, L2 ou L3) est identique chez les moutons et les chèvres (Chi-2 $=0,09, \mathrm{P}>0,05)$.

\section{COMPARAISON DES POPULATIONS CELLULAIRES ENTRE ANIMAUX INFESTÉS ET NON INFESTÉS}

Chez les moutons, de très fortes différences entre individus infestés et individus non infestés sont notées (tableau II), en particulier pour les mastocytes muqueux dans le sinus ainsi que pour les éosinophiles dans les cornets, le sinus et le septum. Dans l'analyse factorielle discriminante, $100 \%$ des moutons infestés ont une réponse cellulaire de type "mouton infesté " et tous les moutons indemnes ont des caractéristiques cellulaires de type "mouton indemne ". La réponse éosinophilique est très variable selon les individus et il n'y a pas de globules leucocytes chez le mouton non infesté.

Chez les chèvres, les différences entre individus indemnes et individus parasités sont très significatives pour chacune des douze variables en particulier pour les mastocytes séreux dans les cornets, le septum et les sinus et pour les éosinophiles dans le septum et les sinus (tableau II). L'AFD indique que toutes les chèvres présentent des réactions cellulaires conformes à leur statut parasitaire (infesté ou non). La grande dis-

\begin{tabular}{|c|c|c|c|c|c|c|}
\hline Espèce hôte & $\begin{array}{l}\text { Nombre } \\
\text { d'animaux } \\
\text { examinés }\end{array}$ & $\begin{array}{l}\text { Nombre } \\
\text { d'animaux } \\
\text { porteurs }\end{array}$ & $\begin{array}{c}\text { Intensité } \\
\text { moyenne } \\
\text { (larves/animal parasité) }\end{array}$ & $\begin{array}{l}\% \text { de larves } \\
\text { de premier } \\
\text { stade }(\mathrm{L} 1)\end{array}$ & $\begin{array}{l}\% \text { de larves } \\
\text { de deuxième } \\
\text { stade (L2) }\end{array}$ & $\begin{array}{l}\text { \% de larves } \\
\text { de troisième } \\
\text { stade ((L3) }\end{array}$ \\
\hline Chèvres & 47 & 23 & 5,34 & 88,6 & 11,3 & 0,01 \\
\hline Moutons & 60 & 30 & 17,3 & 92,1 & 3,85 & 4,05 \\
\hline
\end{tabular}

Tableau I. - Populations parasitaires présentes au moment de l'autopsie sur l'échantillon de moutons et de chèvres.

\begin{tabular}{|c|c|c|c|c|c|c|c|}
\hline \multirow[b]{2}{*}{$\begin{array}{l}\text { Localisation } \\
\text { anatomique }\end{array}$} & \multirow[b]{2}{*}{ Cellule } & \multicolumn{3}{|c|}{$\begin{array}{l}\text { Moutons } \\
(\mathrm{N}=60)\end{array}$} & \multicolumn{3}{|c|}{$\begin{array}{l}\text { Chèvres } \\
(\mathrm{N}=47)\end{array}$} \\
\hline & & infestés & non infestés & $\begin{array}{l}\text { Valeur de } \\
\mathrm{F}(1 / 58)\end{array}$ & infestées & $\begin{array}{c}\text { non } \\
\text { infestées }\end{array}$ & $\begin{array}{c}\text { Valeur de } \\
\mathrm{F}(1 / 45) \\
\end{array}$ \\
\hline \multirow[t]{4}{*}{ Sinus } & Mastocytes séreux & $48(7,6)$ & $33(6,5)$ & $51^{* * * *}$ & $35,5(5,3)$ & $24,8(2,3)$ & $94^{* * *}$ \\
\hline & Mastocytes muqueux & $79(14)$ & $46,5(5,2)$ & $179^{* * *}$ & $44,4(6,1)$ & $33,8(3,7)$ & $51,5^{* * *}$ \\
\hline & Eosinophiles & $152,5(122)$ & $7,6(10,7)$ & $138^{* * *}$ & $67,2(51,3)$ & $7(6,7)$ & $83^{* * *}$ \\
\hline & Globules leucocytes & $12,3(8,3)$ & 0 & $169^{* * * *}$ & $10,3(6)$ & $3(4,2)$ & $29,5^{* * *}$ \\
\hline \multirow[t]{4}{*}{ Cornets } & Mastocytes séreux & $49,4(10,4)$ & $32(9,5)$ & $35^{* * *}$ & $31,5(5,2)$ & $21(2,7)$ & $89^{* * *}$ \\
\hline & Mastocytes muqueux & $73,3(16,2)$ & $43,7(9)$ & $81^{* * *}$ & $37,3(4,1)$ & $27,6(3,7)$ & $66^{* * *}$ \\
\hline & Eosinophiles & $77(41)$ & $6,5(9,5)$ & $145^{* * *}$ & 32,2 (18) & $6,4(8)$ & $57^{* * *}$ \\
\hline & Globules leucocytes & $7,7(7,7)$ & 0 & $65^{* * *}$ & $7,5(5,4)$ & $1,8(3,5)$ & $23^{* *}$ \\
\hline \multirow[t]{4}{*}{ Septum } & Mastocytes séreux & $40,3(9,5)$ & $27(8,3)$ & $27^{* * * *}$ & $25,6(3,3)$ & $16,5(2,3)$ & $132^{* * *}$ \\
\hline & Mastocytes muqueux & 64,3 (11) & $39,2(7,6)$ & $98^{* * *}$ & $30,5(5)$ & $24,2(3)$ & $27^{* * *}$ \\
\hline & Eosinophiles & $58(38,2)$ & $4(3,7)$ & $122 * * *$ & $27,8(15,5)$ & $4,2(3,8)$ & $84^{* * *}$ \\
\hline & Globules leucocytes & $4,7(4)$ & 0 & $73^{* * * *}$ & $4,8(2,8)$ & $0,75(1,1)$ & $45,8^{* * *}$ \\
\hline
\end{tabular}

** $\mathrm{P}<0,01$

*** $\mathrm{P}<0,001$.

Tableau II. - Moyennes (écart-types) des dénombrements de mastocytes, d'éosinophiles et de globules leucocytes chez les moutons et des chèvres infestées et non infestées par CEstrus ovis et valeurs de F associées (analyse de variance). 
persion des réponses éosinophiliques est également très importante. Contrairement aux moutons, il a été possible de mettre en évidence quelques rares globules leucocytes chez les chèvres non infestées.

\section{DIFFÉRENCES DE RÉACTIONS CELLULAIRES ENTRE MOUTONS ET CHEVRES}

Compte tenu de la différence d'intensité d'infestation entre moutons et chèvres, nous avons étudié un sous ensemble de "moutons " excluant les individus à fortes charges parasitaires pour ne plus avoir de différence significative dans l'intensité de l'infestation des moutons et des chèvres.

Comparaison chèvre/moutons chez les individus indemnes (tableau III)

Le nombre d'éosinophiles ne diffère pas entre moutons et chèvres quelle que soit la localisation anato- mique mais en revanche, les mastocytes et particulièrement les mastocytes muqueux sont beaucoup plus nombreux chez les moutons que chez les chèvres. L'AFD donne cette fois un pourcentage de bien classés de $94,4 \%$, un mouton ayant des caractéristiques cellulaires de chèvres et deux chèvres ayant des caractéristiques du groupe " mouton" (tableau IV).

Comparaison chèvre/mouton chez les individus parasités (tableau III)

On retrouve cette différence de réaction mastocytaire chez les individus infestés, en particulier pour les mastocytes muqueux. En revanche, il n'y a plus de différences significatives entre ovins et caprins pour les globules leucocytes et pour les éosinophiles dans le septum. L'AFD montre que toutes les chèvres infestées ont un profil de réactivité cellulaire de type chèvre et que tous les moutons ont un profil de réactivité de type

\begin{tabular}{|c|c|c|c|c|c|c|c|}
\hline \multirow[b]{2}{*}{$\begin{array}{l}\text { Localisation } \\
\text { anatomique }\end{array}$} & \multirow[b]{2}{*}{ Cellule } & \multicolumn{3}{|c|}{$\begin{array}{c}\text { Non infestês } \\
(N=54)\end{array}$} & \multicolumn{3}{|c|}{$\begin{array}{l}\text { Infestés } \\
(\mathrm{N}=46)\end{array}$} \\
\hline & & Chèvres & Moutons & $\begin{array}{c}\text { Valeur de } \\
\mathrm{F}(1 / 52)\end{array}$ & Chèvres & Moutons & $\begin{array}{c}\text { Valeur de } \\
F(1 / 44)\end{array}$ \\
\hline \multirow[t]{4}{*}{ Sinus } & Mastocytes séreux & $24,8(2,3)$ & $33,1(6,5)$ & $27,7^{* * *}$ & $35,5(5,3)$ & $47,4(7,8)$ & $36^{* * *}$ \\
\hline & Mastocytes muqueux & $33,8(3,7)$ & $46,5(5,25)$ & $95^{* * *}$ & $44,3(6,1)$ & $75,8(11,5)$ & $142,7^{* * *}$ \\
\hline & Éosinophiles & $7(6,7)$ & $7,6(10,7)$ & $0,83 \mathrm{NS}$ & $67,2(51,3)$ & $162,5(134)$ & $5,88^{* *}$ \\
\hline & Globules leucocytes & $3(4,2)$ & 0 & $13,22^{* * *}$ & $7(0,96)$ & $6,9(1,66)$ & $0,51 \mathrm{NS}$ \\
\hline \multirow[t]{4}{*}{ Cornets } & Mastocytes séreux & $21(2,7)$ & $32,1(9,5)$ & $22^{* * *}$ & $31,5(5,2)$ & $46,9(8,1)$ & $61^{* * *}$ \\
\hline & Mastocytes muqueux & $27,6(3,7)$ & $43,7(9)$ & $65,7^{* * *}$ & $37,3(4,1)$ & $69,6(13,8)$ & $162,7^{* * *}$ \\
\hline & Eosinophiles & $6,4(8)$ & $6,5(9,5)$ & 0,7 NS & $32,2(18)$ & $76,4(44,4)$ & $13,28^{* *}$ \\
\hline & Globules leucocytes & $0,82(1,26)$ & 0 & $15,5^{* * *}$ & $5,2(1,47)$ & $3,26(1,83)$ & $1,6 \mathrm{NS}$ \\
\hline \multirow[t]{4}{*}{ Septum } & Mastocytes séreux & $16,5(2,3)$ & $27(8,3)$ & $29,5^{* * *}$ & $25,7(3,3)$ & $39,5(9,5)$ & $46^{* * *}$ \\
\hline & Mastocytes muqueux & $24,2(3)$ & $39,2(7,6)$ & $84,4^{* * *}$ & $30,5(5)$ & $62,6(11)$ & $193^{* * *}$ \\
\hline & Eosinophiles & $4,2(3,8)$ & $3,9(3,7)$ & $0,47 \mathrm{NS}$ & $27,8(15,5)$ & $55,7(40,5)$ & $3,15 \mathrm{NS}$ \\
\hline & Globules leucocytes & $0,46(0,75)$ & 0 & $13,2^{* * *}$ & $3,9(0,9)$ & $2,7(1,5)$ & $1,5 \mathrm{NS}$ \\
\hline
\end{tabular}

** $\mathrm{P}<0,01$.

$* * * * \mathrm{P}<0,001$.

Tableau III. - Comparaison des populations cellulaires des moutons et des chèvres lors d'infestations par Estrus ovis d'intensité égales et en absence d'infestations patentes au moment de l'abattage (analyse de variance).

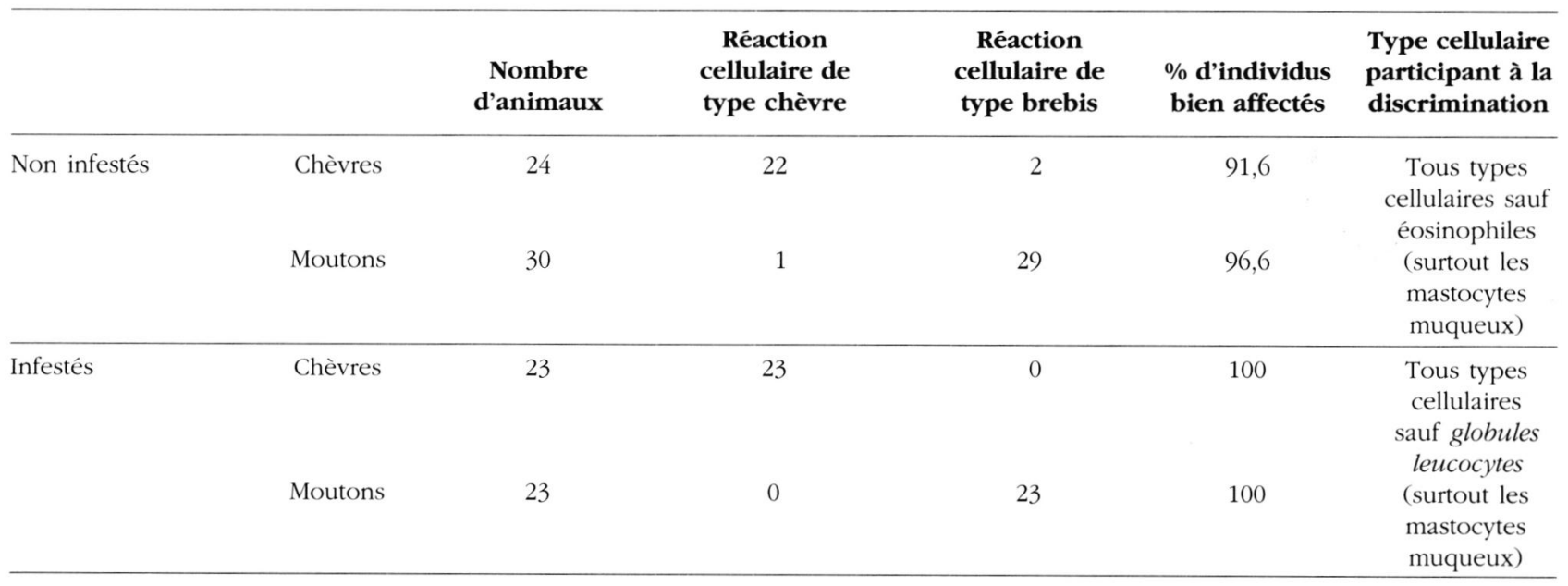

Tableau IV. - Identification des groupes " moutons " et " chèvres " et classement des individus par l'analyse factorielle discriminante. 
mouton (tableau IV). Le type cellulaire qui participe le plus efficacement à cette discrimination totale est le mastocyte muqueux. Il apparait donc qu'au même moment de l'année, pour des charges larvaires identiques, moutons et chèvres développent des réactions cellulaires différentes sur les muqueuses nasales et sinusales face à l'infestation par $O$. ovis et que l'essentiel de cette différence provient des mastocytes muqueux beaucoup plus présents chez le mouton que chez la chèvre.

\section{RELATION ENTRE L'INTENSITÉ DE LA RÉPONSE} CELLULAIRE ET LE NOMBRE DE LARVES PRÉSENTES

Analyse factorielle des correspondances multiples (figures 1 et 2)

Chez les chèvres comme chez les moutons, les fortes réactions cellulaires sont associées à des charges parasitaires élevées, de même, les très faibles réactions cellulaires sont associées à l'absence de larves. Ces relations sont particulièrement nettes pour les globules leucocytes et les éosinophiles, plus lâches pour les mastocytes sauf dans les fortes infestations. On notera l'homogénéité des réponses dans le septum, les cornets et le sinus pour un type cellulaire donné : les classes de fortes réponses éosinophiliques ou en globules leucocytes dans les sinus (SI 3), les cornets (CO 3) et le septum (SE 3) sont très agrégées sur le graphique plan.

\section{Régression multiple}

La combinaison de trois variables (globules leucocytes dans les sinus, globules leucocytes dans les cornets et éosinophiles dans le septum) suffit pour expliquer $90 \%$ de la variabilité de la variable "larve " chez les moutons $\left(r^{2}=0,91\right.$ pour $R$ multiple maximal $\left.=0,95\right)$. Chez les chèvres, la combinaison de quatre variables (mastocytes séreux dans le sinus, éosinophiles dans le sinus, globules leucocytes dans le septum et globules leucocytes dans les cornets) permet d'expliquer $86 \%$ de la variabilité de la variable "larve " $\left(\mathrm{r}^{2}=0,86\right.$ pour $\mathrm{R}$ multiple maximal de 0,93).

Quel que soit le type d'analyses, on retrouve la corrélation positive forte entre charge parasitaire et intensité de la réponse en éosinophiles et en globules leucocytes.

\section{DISCUSSION}

L es moutons et les chèvres de cette étude étaient des adultes de deux ans et plus, élevés au pâturage donc soumis au risque d'infestations naturelles par Estrus ovis. Il semble difficile qu'au cours de leur vie, les animaux indemnes de cette étude n'aient pas été du tout en contact avec ce parasite très fréquent dans les zones considérées (sud de la France). Dès lors, il convient de considérer les non porteurs de larves à l'autopsie comme des sujets dont le statut reste inconnu. Ils peuvent avoir subi des infestations lors de l'été ou l'automne précédent suivies de l'expulsion des dernières larves de troisième stade avant l'abattage. Il peut s'agir aussi d'animaux présentant une certaine résistance à l'implantation de nouvelles larves de premier stade à l'automne. En dépit de ces réserves, les numérations d'éosinophiles, de mastocytes muqueux ou séreux et de globules leucocytes sont augmentées de façon très significative chez les animaux infestés par rapport aux non infestés. Le classement des individus par AFD (100 \% de moutons et chèvres bien classés) démontre que la présence ou l'absence de larves détermine le profil de réaction cellulaire des muqueuses respiratoires supérieures. Cette différentiation totale provient pour une large part d'une très forte mobilisation des éosinophiles. L'infestation expérimentale unique ou répétitive de chevreaux et d'agneaux par des larves de premier stade d'O. ovis s'accompagne d'un recrutement de mastocytes et d'éosinophiles (Dorchies et al., 1998; Duranton, 1997) et cela conforte les résultats obtenus dans les infestations naturelles. L'absence de globules leucocytes dans les muqueuses respiratoires supérieures des moutons indemnes est en accord avec ce qui a été relevé dans les muqueuses digestives d'agneaux sans parasites gastro-intestinaux (Sommerville, 1956; Huntley et al., 1995). La grande variabilité individuelle dans les réponses cellulaires en particulier des éosinophiles a déjà été observée chez des agneaux infestés par le nématode Nematodirus battus (Winter et al., 1997).

Les infestations par des nématodes gastro-intestinaux sont associées au développement de réactions d'hypersensibilité immédiate mettant en jeu des immunoglobulines E (Jarret \& Miller, 1982; Miller, 1984, 1996a). Ces réactions, qui s'accompagnent d'un recrutement important de mastocytes et d'éosinophiles, ont également été suspectées (Dorchies et al., 1998) lors de l'infestation par $O$. ovis. Le recrutement de cellules inflammatoires dans les muqueuses respiratoires en contact avec des larves d'un diptère myiasigène pose la question du rôle de ces cellules dans l'interaction hôte-parasite et plus particulièrement dans la limitation de la charge parasitaire (limitation de nouvelles infestations, mortalité accrue des larves présentes) ou dans la vitesse de développement des larves. La période durant laquelle s'est déroulée l'étude (janvier et fèvrier) exclut la possibilité de nouvelles infestations (les mouches adultes ne sont actives que de la fin du printemps au début de l'automne), en revanche, c'est à ce moment que se déroulent les premières migrations des L1 vers les cavités sinusales et les mues L1-L2 (Yilma 


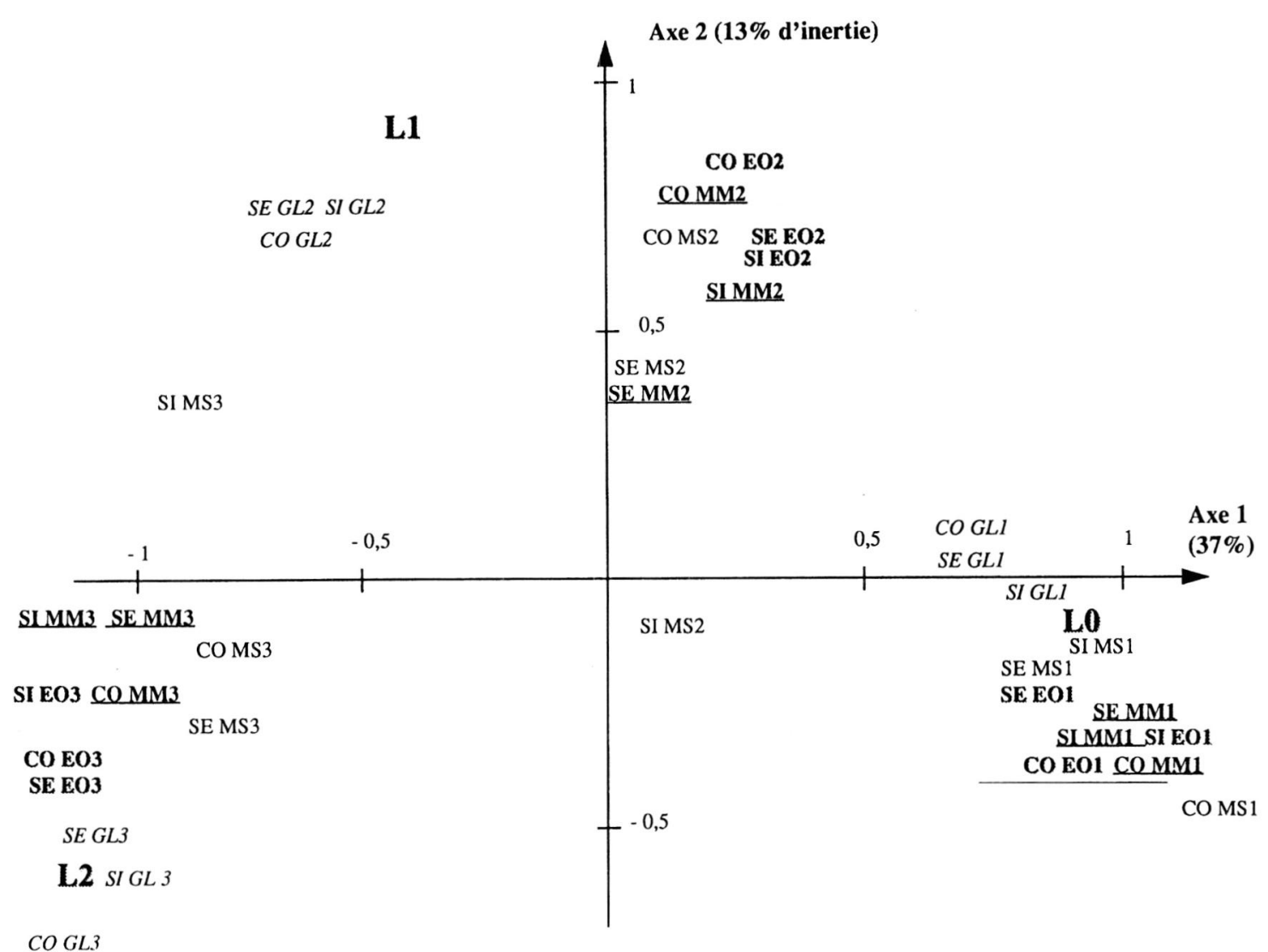

Descriptif des classes

Variable

Larve

Eosinophiles

dans le sinus

Eosinophiles

dans les cornets

Eosinophiles

dans le septum

Globules leucocytes

dans le sinus

Globules leucocytes

dans les cornets
Désignation sur graphique Définition

Lo

L1

L2

SI EO1

SI EO2

SI EO3

CO EO1

$\mathrm{CO} \mathrm{EO} 2$

$\mathrm{CO} \mathrm{EO} 3$

SE EO1

SE EO2

SE EO3

SI GL1

SI GL2

SI GL3

CO GL1

CO GL2

$\mathrm{CO}$ GL3

0 à 5

6 à 67

68 à 438

0 à 4

5 à 56

57 à 163

0 à 4

5 à 40

41 à 153

0

1 à 13

14 à 32

0

1 à 9

10 à 34
Globules leucocytes

dans le septum

pas de larves

1 à 11 larves

12 à 68 larves

Mastocytes séreux

dans le sinus

dans le sinus

Mastocytes séreux

dans les cornets

dans les cornets

Mastocytes séreux

dans le septum

dans le septum
$S E G L 1$

SE GL2

SE GL3

SI MS1

SI MS2

SI MS3

Mastocytes muqueux

Mastocytes muqueux

Mastocytes muqueux
SI MM1

SI MM2

SI MM3

CO MS1

CO MS2

CO MS3

CO MM1

CO MM2

CO MM3

SE MS1

SE MS2

SE MS3

SE MM1

SE MM2

SE MM3
0

1 à 4

5 à 14

17 à 35

36 à 45

46 à 65

31 à 48

49 à 68

69 à 111

9 à 33

34 à 43

44 à 72

22 à 49

50 à 65

66 à 115

8 à 28

29 à 39

40 à 58

19 à 41

42 à 58

59 à 87

Fig. 1. - Relation entre l'intensité de la charge parasitaire et l'intensité de la réponse cellulaire chez les moutons (analyse factorielle des correspondances multiples). 


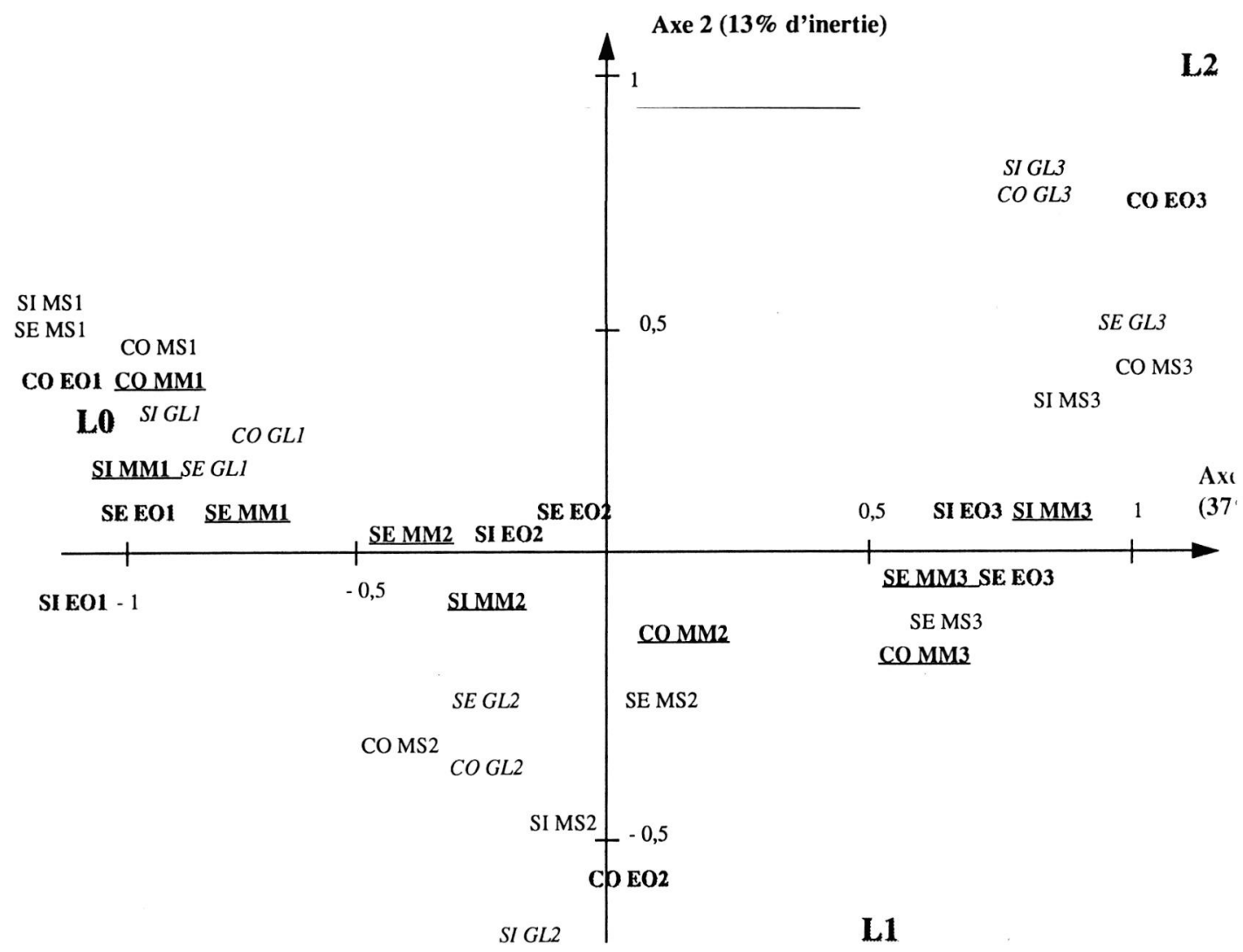

Descriptif des classes

Variable

Larve

Eosinophiles

dans le sinus

Eosinophiles dans les cornets

Eosinophiles

dans le septum

Globules leucocytes

dans le sinus

Globules leucocytes

dans les cornets

\section{Désignation sur graphique}

LO

L1

L2

SI EO1

SI EO2

SI EO3

CO EO1

$\mathrm{CO} \mathrm{EO} 2$

$\mathrm{CO} \mathrm{EO3}$

SE EO1

SE EO2

SE EO3

SI GL1

SI GL2

SI GL3

CO GL1

CO GL2

CO GL3

$\begin{aligned} & \text { Globules leucocytes } \\ & \text { dans le septum }\end{aligned}$
$\begin{aligned} & \text { Mastocytes séreux } \\ & \text { dans le sinus }\end{aligned}$
Mastocytes muqueux
dans le sinus
Mastocytes séreux
dans les cornets
Mastocytes muqueux
dans les cornets
Mastocytes séreux
dans le septum

Globules leucocytes

SE GL1

SE GL2

SE GL3

Définition

pas de larves

1 à 3 larves

4 à 22 larves

1 à 5

6 à 38

39 à 201

1 à 4

5 à 27

28 à 74

1 à 3

4 à 20

21 à 65

0 à 2

3 à 8

9 à 23

0

1 à 5

6 à 18

SI MS1

SI MS2

SI MS3
2

27 à 32

33 à 47

SI MM1 26 à 35

SI MM2 36 à 41

SI MM3 42 à 57

CO MS1 17 à 21

CO MS2 22 à 29

CO MS3 30 à 46

CO MM1 20 à 28

CO MM2 29 à 36

CO MM3 $\quad 37$ à 47

SE MS1 13 à 16

SE MS2 17 à 24

SE MS3 25 à 34

SE MM1 18 à 24

$\underline{\text { SE MM2 }} 25$ à 28

SE MM3 29 à 42

Fig. 2. - relation entre l'intensité de la charge parasitaire et l'intensité de la réponse cellulaire chez les chèvres (analyse factorielle des correspondances multiples). 
\& Dorchies, 1991). Dès lors, les réactions cellulaires enregistrées pendant cette période sont des réactions consécutives à la présence de larves de premier stade en hypobiose ou à celle de larves de deuxième stade beaucoup plus mobiles et physiologiquement actives. Face à ces parasites, les mastocytes mobilisés peuvent agir à plusieurs niveaux : i) libération de médiateurs inflammatoires préformés (histamine, protéoglycannes) ou néoformés dont les leucotriènes C4, D4 et E4 impliqués dans l'inhibition de la migration des larves de nématodes gastrointestinaux chez le mouton (Douch et al., 1993; Douch et al., 1995), ii) augmentation de la perméabilité de la muqueuse notamment par l'intermédiaire de protéases secrétées par les mastocytes et les globules leucocytes (SMCP: sheep mast cell protease, Miller, 1996b) ou de cytokines comme le TNF-alpha qui favorise le passage de protéines plasmatiques (dont des immunoglobulines) dans la lumière intestinale ou les cavités respiratoires et iii) secrétion de cytokines dont les interleukines Il-4, Il-5, Il-6 et Il-10 qui influencent la polarisation de la réponse immunitaire locale dans un sens TH2 (Urban et al., 1996; Wilson, 1993) et augmentent la production d'immunoglobulines d'isotype A (Ramsay et al., 1994). Dans le même temps, les éosinophiles recrutés et activés peuvent agir directement sur les parasites (Tagboto, 1995) par l'intermédiaire de secrétions toxiques (ECP : eosinophil cationic protein; MBP: major basic protein...). La dégranulation des éosinophiles s'accompagne d'une mortalité rapide des larves de premier stade d'O. ovis maintenues in vitro (Duranton, 1997), son mécanisme n'est pas connu dans le cas de l'œestrose mais si l'on se réfère à d'autres modèles (Schistosoma mansoni), ce pourrait être une cytotoxicité dépendante d'anticorps (Butterworth et al., 1975).

La plupart des mécanismes effecteurs sont communs aux chèvres et aux moutons, il existe cependant des différences quantitatives importantes dans le recrutement des cellules inflammatoires (réponse de type " chèvre " et réponse de type "mouton ") face à l'infestation par des larves d'O. ovis). Cette différence a également été mise en évidence par Huntley et al. (1995) dans les modèles Teladorsagia circumcincta et Trichostrongylus vitrinus, deux trichostrongles de petits ruminants respectivement de la caillette et de l'intestin grêle. Chez la chèvre, ces auteurs notent une prépondérance de globules leucocytes et une relative pauvreté en mastocytes muqueux par rapport aux moutons, ces derniers développent au contraire une très forte réaction mastocytaire ainsi que des concentrations élevées en SMCP. En revanche, il ne semble pas y avoir de différence pour les éosinophiles. Dans notre étude, en dehors d'une absence de différence significative pour les globules leucocytes chez les animaux infestés, chèvres et moutons se distinguent par leurs réponses en mastocytes muqueux, séreux et en éosinophiles dans les cornets nasaux et les sinus : cette réponse est beaucoup plus accentuée chez les moutons que chez les chèvres à la même période de l'année à charge parasitaire égale.

Dans les infestations expérimentales d'agneaux par Teladorsagia circumcincta, Stevenson et al. (1994) ont montré une relation inverse entre intensité de la réponse inflammatoire locale (éosinophiles et mastocytes) et nombre de vers récoltés à l'autopsie. Il semble que ce soit rigoureusement l'inverse dans le cas de l'œestrose : les moutons, dont les réactions cellulaires sont intenses, sont plus souvent et plus massivement infestés par O. ovis que les chèvres qui développent une réaction cellulaire plus faible. Cette apparente contradiction est retrouvée dans les infestations expérimentales de chevreaux et d'agneaux par des larves de premier stade d'Estrus ovis: les agneaux développent de plus fortes réactions en mastocytes et en éosinophiles que les chevreaux mais ce sont ces derniers qui, in fine, hébergent moins de larves que les agneaux (Duranton, 1997).

Les différences chèvres et moutons se retrouvent également chez les individus indemnes au moment de l'abattage. Il n'y a pas de différence significative dans le dénombrement des éosinophiles (le nombre de ces cellules diminue rapidement après l'interruption de l'infestation parasitaire, Buddle et al., 1992) mais une différence significative existe pour les mastocytes en particulier muqueux. Ainsi, chez le mouton, les muqueuses du septum nasal, des cornets et des sinus semblent infiltrées par un plus grand nombre de mastocytes que chez les chèvres, et ce, en l'absence de larves d'O. ovis.

En conclusion, face à l'agression parasitaire par les larves d'CEstrus oris, les muqueuses respiratoires supérieures des brebis et des chèvres répondent par un recrutement d'éosinophiles et de mastocytes et leurs dérivés, les globules leucocytes (Jarret et al., 1967; Miller et al., 1967). Il existe une réponse de type chèvre et une réponse de type mouton, se différenciant essentiellement par une plus forte mobilisation des mastocytes muqueux chez les moutons que chez les chèvres.

Cette réponse inflammatoire semble échouer dans le contrôle des larves d' Estrus ovis mais elle pourrait être responsable de la pathogénicité parasitaire. De même, les différences de réponse inflammatoire entre moutons et chèvres seraient à l'origine des différences d'expression clinique de l'œstrose.

\section{REMERCIEMENTS}

es auteurs remercient Messieurs les Professeurs Cabanié et Delverdier du Service d'Anatomie Pathologique de l'École Nationale Vétérinaire de 
Toulouse pour leur aide précieuse dans l'élaboration de ce manuscrit ainsi que l'ensemble du personnel technique de ce Service.

\section{BIBLIOGRAPHIE}

Belem A.M.G. \& Roullle D. CEstrose des petits ruminants au Burkina Faso. Revue d'Elevage et de Médecine Vétérinaire des Pays Tropicaux, 1988, 41(1), 59-64.

Bergeaud J.P., Duranton C. \& Dorchies P. L'œestrose ovine en Aveyron : résultat d'une enquête sur 1036 têtes à l'abattoir de Rodez. Revue de Médecine Vétérinaire, 1994, 145 (11), 863-866.

Buddle B.M., Jowett G., Green R.S., Douch P.G.C. \& Risdon P.L. Association of blood eosinophilia with the expression of resistance in Romney lambs to nematodes. International Journal for Parasitology, 1992, 22, 955-960.

Butterworth A.E., Sturrock R., Houba V., Mahmoud A., SHer A. \& ReEs P. Eosinophils as mediators of antibody dependant damage of Schistosomula. Nature, 1975, 256, 727-729.

Chlabra M.B. \& Ruprah N.S. Observations on the incidence and biology of CEstrus ovis Linné, 1761. Indian Veterinary Journal, 1976, 53 (3), 180-184.

Dorchies P., Yilma J.M. \& Savey J. Prevalence of lung abscesses and interstitial pneumonia in ovine ostrosis. Veterinary Record, 1993, 133, 325.

Dorchies P., Duranton C. \& Jacquiet P. Pathophysiology of Estrus ovis infection in sheep and goats: review. Veterinary Record, 1998, 142, 487-489.

Douch P.G.C., Harrison G.B.L., Buchanan L.L. \& Greer K.S. In vitro bioassay of sheep gastrointestinal mucus for nematode paralysing activity mediated by substances with some properties characteristics of SRA-A. International Journal for Parasitology, 1993, 13, 207-212.

Douch P.G.C., Morum P.E. \& Rabel B. Secretion of anti-parasite substances and leucotrienes from ovine gastrointestinal tissues and isolated mucosal mast cells. International Journal for Parasitology, 1995,26, 205-211.

Duranton C. Comparaison de l'infestation par CEstrus ovis (Linné, 1761) chez la chèvre et le mouton. Thèse de l'Université Paul Sabatier de Toulouse, 1997, 208 p.

Horak G. Parasites of domestic and wild animals in South Africa. I. Estrus ovis in sheep. Onderstepoort Journal of Veterinary Research, 1977a, 44 (2), 55-64.

Horak G. Parasites of domestic and wild animals in South Africa. II. CEstrus ovis in goats. Onderstepoort Journal of Veterinary Research, 1977b, 44 (2), 65-68.

Huntley J.F., Patterson M., MacKellar A., Jackson F., SteVENSON L.M. \& COOP R.L. A comparison of the mast cell and eosinophil responses of sheep and goats to gastrointestinal nematode infections. Research in Veterinary Science, 1995, 58, 5-10.

Jagannah M.S., Nurella C. \& Vijayasarathi S.K. Histological changes in the nasal passages of sheep and goats infested with CEstrus ovis (Diptera: (Estridae). Indian Journal of Animal Science, 1989, 59 (1), 87-91.
Jarrett W.F.H., Miller H.R.P. \& Murray M. The relationship between mast cells and globules leucocytes in parasitic infections. Veterinary Record, 1967, 80 (16), 505-506.

Jarret E.E.E., Miller H.R.P. Production and activities of IgE in helminth infections. Progress in Allergy, 1982, 31, 178233 .

Miller H.R.P. The protective mucosal response against gastrointestinal Nematodes in ruminants and laboratory animals. Veterinary Immunology and Immunopathology, $1984,6,167-259$.

Miller H.R.P. Mucosal mast cells and the allergic response against nematode parasites. Veterinary Immunology and Immunopathology, 1996a, 54, 331-336.

Miller H.R.P. Prospects for the immunological control of ruminant gastrointestinal Nematodes : natural immunity, can it be harnessed? International Journal for Parasito$\log y, 1996 \mathrm{~b}, 26$ (8/9), 801-811.

Miller H.R.P., Murray M. \& JarRet W.F.H. Globule leucocytes and mast cells. In : The reaction of the host to parasitism. Veterinary Medical Review, 1967, 198-210.

Nguyen Van-Khanh, Bourges N., Concordet D. \& Dorchies P. Mastocytes et éosinophiles de la muqueuse respiratoire du mouton infesté par Estrus ovis (Linné, 1761). Parasite, 1996, 3, 217-221

OgunRinade A.F. Preleminary observation on Estrus ovis myiasis in West African Dwarf goats in Nigeria. Bulletin of Animal Health and Production, 1977, 25 (2), 154-156.

Ramsay A.J., Husband A.J., Ramshaw I.A., Bao S., Matthaei K.I., Koenler G. \& Kopf M. The role of interleukin-6 in mucosal IgA antibody response in vivo. Science, 1994, 264, 561-563.

Sommerville R.I. The histology of the ovine abomasum, and the relation of the globule leucocyte to nematode infections. Australian Veterinary Journal, 1956, 32, 237-240.

Stevenson L.M., Huntley J.F., Smith W.D. \& Jones D.G. Local eosinophil and mast cell-related responses in abomasal nematode infections of lambs. Immunology and Medical Microbiololgy, 1994, 8, 167-174.

TAGBOTO S.K. Interleukin-5, eosinophils and the control of helminth infections in man and laboratory animals. Journal of Helminthology, 1995, 69, 271-278.

Urban J.F., Fayer R., Sullivan C., Goldhill J., SheaDonohue T., Madden K., Morris S.C., Katona I., Gause W., Ruff M., Mansfield L.S. \& Finkelman F.D. Local TH1 and TH2 responses to parasitic infection in the intestine: regulation by IFN-gamma and Il-4. Veterinary Immunology and Immunopathology, 1996, 54, 337-344.

WILSON R.A. Immunity and immunoregulation in helminth infections. Current Opinion in Immunology, 1993, 5, 538547.

Winter M.D., Wright C. \& Lee D.L. The mast cell and eosinophil response of young lambs to a primary infection with Nematodirus battus. Parasitology, 1997, 114, 189-193.

Yilma J.M. \& Dorchies P. Epidemiological study of Estrus ovis in south west France. Veterinary Parasitology, 1991, 40, 315-323.

Reçu le 12 novembre 1998 Accepté le 27 janvier 1999 